\title{
Association Between Birth Weight and Adult Disorders in Twins: Validity of Self-reported Birth Weight
}

\author{
Thomas Heiberg Brix', Kirsten Ohm Kyvik², Laszlo Hegedüs ${ }^{3}$ \\ 'Department of Endocrinology, Odense University Hospital, Denmark \\ ${ }^{2}$ The Danish Twin Register, Odense University, Denmark \\ ${ }^{3}$ Department of Endocrinology, Odense University Hospital, Denmark.
}

As was pointed out issue 4.5 of Twin Research, a growing number of studies have suggested that small size at birth, is associated with an increased propensity to a number of adverse health outcomes in adult life, including hypertension and cardiovascular disease (the fetal origins hypothesis) (Roseboom \& Lambalk, 2001). These associations could, however, also be due to genetic or socioeconomic factors affecting both birth weight and health in later life (e.g., size of blood pressure). These confounders can, despite a number of methodological limitations and potential biases, to some extent be controlled for through studies of twin pairs (Leon, 2001).

One very important source of bias not adequately addressed in a number of twin studies concerning birth weight and later disease is the validity of self-reported birth weight data (Leon, 2001). In line with Leon (2001) we think that a measurement of the validity of self-reported birth weight and intra-pair birth weight difference is crucial when interpreting analyses using this type of data.

We had the opportunity to examine the validity of self-reported birth weight and intra-pair birth weight difference in a subsample of same-sex Danish twin pairs born between 1953-1972 (Brix et al., 1999). In 302 twin individuals (139 complete pairs) we had information on both self-reported and measured birth characteristics. Self-reported birth weight and birth order (first vs. second born) was obtained from a questionnaire survey, whereas measured birth weight was obtained from the original midwife protocols. Classification of the twins in a pair as the first- or second-born twin was based on information from the participating twins concerning the birth sequence. There was complete agreement concerning individual birth sequences within all twin pairs when asking each twin separately. The degree of agreement between the two measures of birth weight can be estimated by using a simple plot of the difference between the two methods (self-reported minus measured birth weight) against their mean (self- reported + measured birth weight divided by 2) (Bland \& Altman, 1986) (see Figure). From our data, the mean difference in birth weight (self-reported - measured) is 6.2 gram with a standard deviation of 340 gram, giving a $95 \%$ confidence interval of -660 to +672 gram. Thus, the self-reported birth weight may be 660 gram below or 672 gram above the measured birth weight, (see Figure).

In a similar way we estimated the validity of the intra-pair birth weight difference (birth weight of the first minus second born) when birth

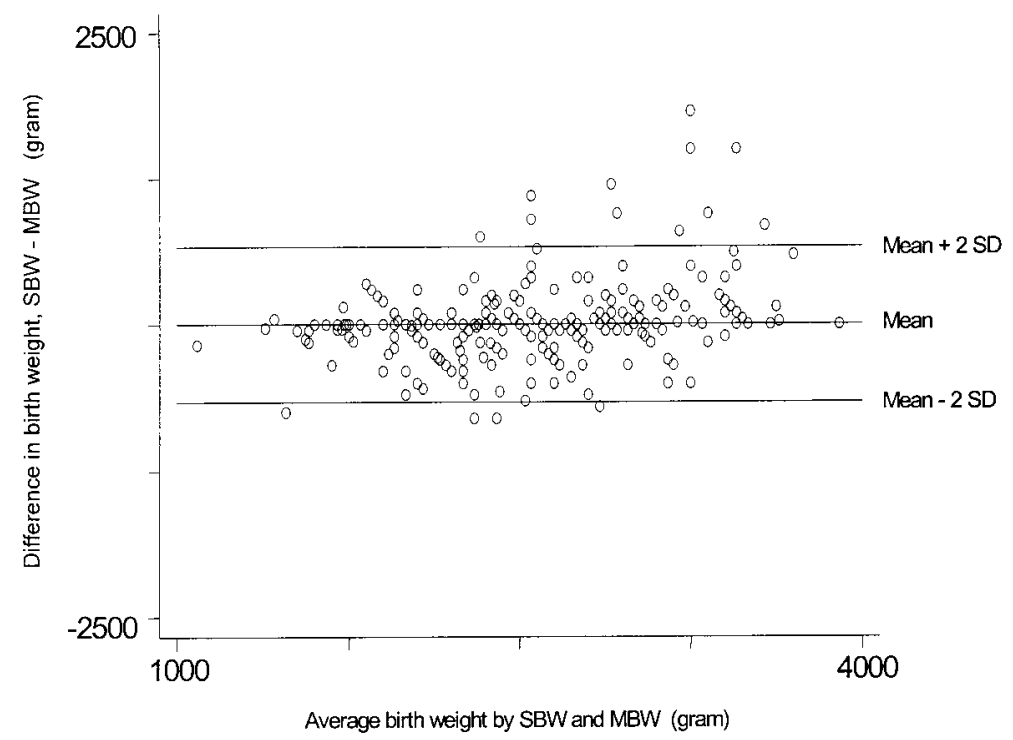

SBW = self-reported birth weight, $\mathrm{MBW}=$ measured birth weight, $\mathrm{SD}=$ standard deviatiov

Figure 1

Average birth weight by SBW and MBW.

Address for correspondence: Thomas Heiberg Brix, Department of Endocrinology, Odense University Hospital, Denmark. Email: TBrix@health.sdu.dk 
weight was self-reported. Among the complete 139 twin pairs, the mean difference in intra-pair birth weight difference was -24 gram ( $95 \%$ confidence interval; -863 gram to +815 gram). Although, the mean differences between self-reported and measured birth weight/intra-pair birth weight difference is small (6.2 gram), the confidence intervals are very wide, and reflect that there are considerable discrepancies between the two measurements of birth weights. We suggest that validated birth characteristics should be used for this type of study.

\section{References}

Bland, J. M., \& Altman, D. G. (1986). Statistical methods for assessing agreement between two methods of clinical measurement. Lancet, I, 307-310.
Brix, T. H., Kyvik, K. O., \& Hegedüs, L. (2000). Low birth weight is not associated with clinically overt thyroid disease: A population based twin case-control study. Clinical Endocrinology, 53, 17I-176.

Leon, D. A. (200I). The foetal origins of adult disease: Interpreting the evidence from twin studies. Twin Research, 4, 321-326.

Roseboom, T. J., \& Lambalk, C. B. (200I). The fetal origins hypothesis. Twin Research, 4, 3.

\title{
Birthweight in Dizygotic Twins
}

\author{
William H. James \\ The Galton Laboratory, University College of London, UK
}

Loos et al. (2001) reported that among a large sample $(n=1929)$ of Dutch dizygotic (DZ) twin pairs, mean length of gestation in MF pairs is similar to that in FF pairs, and significantly longer than in MM pairs. Mean birthweight of girls from MF pairs was similar to that of girls in FF pairs, but boys from MF pairs were significantly heavier than boys from MM pairs. These authors concluded that "these data show that in unlikesex pairs, it is the girl that prolongs gestation for her brother". I responded (James, 2002) with the suggestion that differences in birthweight are consequent on competition for nutrient, and that males, being programmed to grow faster, are more successful in that competition. I acknowledged that my interpretation "would carry the expectation that females in MF pregnancies should weigh less than females in FF pregnancies" (a feature which was not evident in the data of Loos et al., 2001). Here I draw attention to some prior data which confirm that suggestion.

Orlebeke et al. (1993) reported on a larger sample of Belgian twins $(n=2277 \mathrm{DZ}$ pairs $)$. In this sample,

Table 1

Mean Birthweights (Grams) of Dizygotic Twin Members by Sex (Data of Orlebeke et al., 1993)

\begin{tabular}{lccc}
\hline & \multicolumn{3}{c}{ Sex Combination of Pair } \\
& $\mathrm{MM}(n=651)$ & $\mathrm{MF}(n=542)$ and FM $(n=490)$ & $\mathrm{FF}(n=594)$ \\
\hline Male & 2600 & 2622 & 2510 \\
Female & & 2484 & 2510 \\
\hline
\end{tabular}

boys from MF pairs weighed slightly more than those from MM pairs: and girls from MF pairs weighed slightly less than those from FF pairs. The mean birthweights of the male and female twin members are given here (see Table 1). This evidence seems to favour the suggestion that the availability of, and competition for, nutrient may have influenced the birthweights of dizygotic twins in the various categories of sex combination.

Bulmer (1970) wrote that "there can be no doubt that a large part of the reduction in the birth weight in multiple births is due to some factor other than shorter length of gestation". He concluded (p. 52) that the factor is probably an insufficient blood supply to the uterus. In other words, there is competition for nutrient among the occupants of the uterus. The notion that, within a litter, large litter-members grow large at the expense of small ones, has also been suggested in respect not only of man, but of other mammalian species, namely, the sheep and the rabbit (Beatty, 1956).

\section{References}

Beatty, R.A. (1956). Relation between genetic constitution of an offspring and weight of its litter-mates. Nature, I 78, 48.

Bulmer, M. G. (1970). The biology of twinning in man (p. 48). Oxford: Clarendon Press.

James, W. H. (2002). Gestation and birthweight in dizygotic twins. Lancet, 359 , $17 \mid-172$.

Loos, R. J. F., Derom, C., Eeckles, R., Derom, R., \& Vlietinck, R. (200I). Length of gestation and birthweight in dizygotic twins. Lancet, 358, 560-56I.

Orlebeke, J. F., van Baal, C. G. M., Boomsma, D. I., \& Neeleman, D. (1993). Birth weight in opposite sex twins as compared to same sex dizygotic twins. European Journal of Obstetrics \& Gynecology and Reproductive Biology, 50, 95-98. 